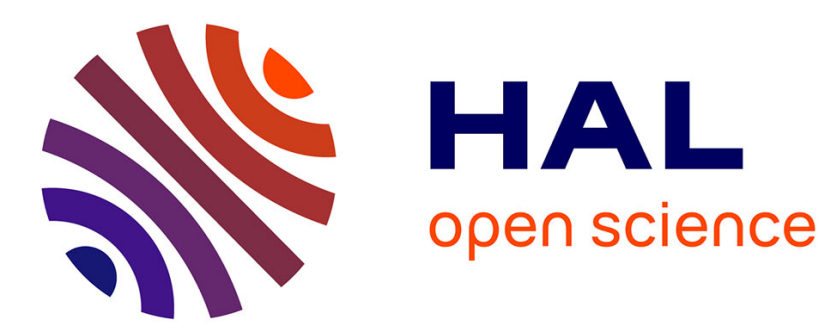

\title{
On the Analysis of Single-Doppler Radar Data
}

P. Waldteufel, H. Corbin

\section{To cite this version:}

P. Waldteufel, H. Corbin. On the Analysis of Single-Doppler Radar Data. Journal of Applied Meteorology, 1979. hal-01923277

\section{HAL Id: hal-01923277 \\ https://hal.uca.fr/hal-01923277}

Submitted on 8 Jun 2021

HAL is a multi-disciplinary open access archive for the deposit and dissemination of scientific research documents, whether they are published or not. The documents may come from teaching and research institutions in France or abroad, or from public or private research centers.
L'archive ouverte pluridisciplinaire HAL, est destinée au dépôt et à la diffusion de documents scientifiques de niveau recherche, publiés ou non, émanant des établissements d'enseignement et de recherche français ou étrangers, des laboratoires publics ou privés. 


\title{
On the Analysis of Single-Doppler Radar Data
}

\author{
P. Waldteufei aNd H. CoRBin \\ Universite de Clermont II, Observatoire du Puy de Dome, 63000 Clermont-Ferrand, France
}

(Manuscript received 15 September 1978, in final form 29 December 1978)

\begin{abstract}
The procedures currently employed to retrieve vector wind information from single-Doppler radar observations are reviewed briefly. In particular, those procedures implying a linearity hypothesis for the wind field are shown to be particular cases of a method termed VVP (Volume Velocity Processing). This method, which makes full use of radar velocity data filling a volume, is first tested on simulated observations in order to assess its accuracy, then applied to actual data and shown to yield unbiased parameters of the horizontal vector wind field, as well as an estimate of the hydrometeor fall velocity and several control parameters.
\end{abstract}

\section{Introduction}

Since Doppler radar were first used in meteorological observations and research, a sizable number of studies has been devoted to the problem of singleDoppler data analysis. Stated briefly, the crux of this problem lies in the fact that one would like to know as much as possible about the wind vector field, whereas a single-Doppler radar yields only one wind component, the radial. Every attempt to gain some knowledge of the vector field, therefore, must compound the data field with additional hypotheses or simplifications.

In a study by Peace et al. (1969), the main hypothesis is that the echo region moves across the radar field of view with little modification of the wind field structure, to such an extent that two radar explorations separated in time can be viewed as a simultaneous exploration by two distant radars. Every other method known to the authors assumes a simplifying property of the wind field itself, rather than its time variation. With the single exception of a study by Harris (1975), who assumed that the mean wind was a harmonic function of space, this simplifying property has been that of linearity. Lhermitte and Atlas (1961) initially suggested the VAD (Velocity Azimuth Display) method and showed how the mean horizontal wind magnitude and direction can be retrieved from radial velocity data around horizontal circles centered along the vertical of the radar site. Other authors (Caton, 1963;'Browning and Wexler, 1968) demonstrated how this concept can be extended to yield such other parameters as mean convergence, stretching and shearing deformation-all this assuming that the wind vector field varies linearly in the horizontal plane. A 0021-8952/79/040532-11\$06.75

(C) 1979 American Meteorological Society study by Easterbrook (1975) was then concerned with processing data in a conical sector rather than along a circle. He showed that indeed five parameters of the wind field can be extracted; however, unless additional information is available, the mean horizontal velocity is contaminated by vorticity and cannot be completely determined.

Here we proceed somewhat further along this line and investigate more generally what can be gained by processing single-Doppler data in a volume, assuming linearity of the wind field. The interest of this analysis is twofold: first, it puts clearly in perspective the processing methods used so far, and brings to light the limitations inherent to every main variation of the linear analysis scheme; second, we find that processing a volume does provide interesting extra information, as demonstrated with actual radar data.

\section{Analysis}

We adopt a Cartesian reference frame $x, y, z$, where the radar is the origin and $z$ the vertical coordinate, and assume the vector representative of the motion of scatterers $\mathbf{V}=(u, v, w)$ varies linearly around its value $\left(u_{0}, v_{0}, w_{0}\right)$ at a point $\left(x_{0}, y_{0}, z_{0}\right)$, i.e.,

$$
u=u_{0}+u_{x}^{\prime}\left(x-x_{0}\right)+u_{y}^{\prime}\left(y-y_{0}\right)+u_{z}^{\prime}\left(z-z_{0}\right) \text {, }
$$

and the same for $v$ and $w$.

The radial velocity $V$, measured by the radar is

$$
V_{r}=-u \cos \theta \cos \phi-v \sin \theta \cos \phi-w \sin \phi,
$$

where $\theta$ and $\phi$ are the azimuth and elevation angles. Transforming from rectangular to polar coordinates, introducing the radial distance $R$ and regrouping 
terms, we obtain

$$
\begin{aligned}
V_{r}= & \cos \theta \cos \phi\left(u_{0}-u_{x}^{\prime} x_{0}-u_{y}^{\prime} y_{0}-u_{z}^{\prime} z_{0}\right) \\
& +\sin \theta \cos \phi\left(v_{0}-v_{x}^{\prime} x_{0}-v_{y}^{\prime} y_{0}-z_{z}^{\prime} z_{0}\right) \\
& +\sin \phi\left(w_{0}-w_{x}^{\prime} x_{0}-w_{y}^{\prime} y_{0}-w_{z}^{\prime} z_{0}\right) \\
& +R \cos ^{2} \theta \cos ^{2} \phi u_{x}^{\prime} \\
& +R \sin ^{2} \theta \cos ^{2} \phi v_{y}^{\prime} \\
& +R \sin \theta \cos \theta \cos ^{2} \phi\left(u_{y}^{\prime}+v_{x}^{\prime}\right) \\
& +R \sin ^{2} \phi w_{z}^{\prime} \\
& +R \cos \theta \sin \phi \cos \phi\left(u_{z}^{\prime}+w_{x}^{\prime}\right) \\
& +R \sin \theta \sin \phi \cos \phi\left(v_{z}^{\prime}+w_{y}^{\prime}\right) \\
= & \sum_{j=1,9} Q_{j} .
\end{aligned}
$$

Since the only way to discriminate between terms of $V_{r}$ is to rely on their different dependence on polar coordinates, the best one can ever hope is to obtain nine independent parameters. Since the full linear wind field is described by 12 parameters, it is already clear that the total retrieval of this field is not possible without further restrictions.

We now accept a minimum number of such restrictions or hypotheses in order that Eq. (3) can be used:

(i) As is well known from previous work and in tuition (see Easterbrook, 1975), horizontal vorticity cannot be extracted from single-Doppler data. The derivatives $u_{y}^{\prime}$ and $v_{x}^{\prime}$ appear either summed together in $Q_{6}$ (yielding the deformation) or with other unknown quantities in $Q_{1}$ and $Q_{2}$. Thus, the only possibility of. retrieving $u_{0}$ and $v_{0}$ in the absence of extra information is to choose $x_{0}=y_{0}=0$.

(ii) In locally stratiform situations where a linear approximation is most likely to apply, it is certainly appropriate to neglect $w_{x}^{\prime}$ and $w_{y}^{\prime}$ with respect to $u_{z}^{\prime}$ and $v_{z}^{\prime}$ in $Q_{8}$ and $Q_{9}$.

We shall come back to these limitations later and see that, on one hand, (i) does not really preclude the use of Eq. (3) in asymmetric volumes, and on the other hand, the validity of (ii) can be checked further in the processing. In any case, we can now rearrange (3) to obtain

$$
\begin{aligned}
V_{r} \approx & \cos \theta \cos \phi u_{0}+\sin \theta \cos \phi v_{0}+\sin \phi w_{0} \\
& +R \cos ^{2} \theta \cos ^{2} \phi u_{x}^{\prime}+R \sin ^{2} \theta \cos ^{2} \phi v_{y}^{\prime} \\
& +R \sin \theta \cos \theta \cos ^{2} \phi\left(u_{y}^{\prime}+v_{x}^{\prime}\right) \\
& +\sin \phi\left(r \sin \phi-z_{0}\right) w_{z}^{\prime} \\
& +\cos \theta \cos \phi\left(R \sin \phi-z_{0}\right) u_{z}^{\prime} \\
& +\sin \theta \cos \phi\left(R \sin \phi-z_{0}\right) v_{z}^{\prime} \\
\approx & \sum_{j=1,9} V_{j} .
\end{aligned}
$$

The retrieval of all nine $V_{r}$ terms from Eq. (4) is now possible.

\section{a. Processing a circle}

This is the original VAD technique. Only terms with different azimuth dependence can be discrimi- nated. Since $R$ and $\phi$ are constant, we can regroup terms in (4) to obtain

$$
\begin{aligned}
V_{r}= & \cos \theta \cos \phi u_{0}+\sin \theta \cos \phi v_{0} \\
& +\cos ^{2} \theta\left[R \cos ^{2} \phi u_{x}^{\prime}+\sin \phi w_{0}\right] \\
& +\sin ^{2} \theta\left[R \cos ^{2} \phi v_{y}^{\prime}+\sin \phi w_{0}\right] \\
& +\sin \theta \cos \theta R \cos ^{2} \phi\left(u_{y}^{\prime}+v_{x}^{\prime}\right) \\
= & \sum_{j=1,5} S_{j} .
\end{aligned}
$$

Terms proportional to $w_{x}^{\prime}$ and $w_{y}^{\prime}$ have again been left aside, since they should stay comparatively small. On the other hand, contamination by the vertical velocity term appearing in $S_{3}$ and $S_{4}$ can be a serious problem, especially in view of computing the horizontal divergence. For this reason, use of the VAD method has generally been limited to low elevations. Several efforts to estimate the vertical component (the bulk of which consists of the fall velocity of hydrometeors) from radar reflectivity data have been made, but the result so far cannot be considered as entirely satisfactory (see, e.g., Battan, 1977).

\section{b. Processing a conical surface}

This method has been proposed by Easterbrook (1975) under the name of VARD (Velocity ARea Display) with application to a conical sector, i.e., $x_{0} \neq 0, y_{0} \neq 0$. When low elevation angles are considered $(\sin \phi \approx 0, \cos \phi \approx 1)$, Eq. (3) becomes

$$
\begin{aligned}
& V_{\tau} \approx \cos \theta\left(u_{0}-u_{x}^{\prime} x_{0}-u_{y}^{\prime} y_{0}\right)+\sin \theta\left(v_{0}-v_{x}^{\prime} x_{0}-v_{y}^{\prime} y_{0}\right) \\
& +R \cos ^{2} \theta u_{x}^{\prime}+R \sin ^{2} \theta v_{y}^{\prime}+R \cos \theta \sin \theta\left(u_{y}^{\prime}+v_{x}^{\prime}\right) .
\end{aligned}
$$

The data are processed using a two-dimensional, linear, least-squares technique. It is seen in Eq. (6) that the divergence and deformation are readily retrieved, as in the VAD procedure. On the other hand, vorticity and mean horizontal wind components are combined in the first two terms in such a way that they cannot be separated unless extra information is available. It is found that differential properties of the horizontal wind field can be estimated within $\pm 0.510^{-3} \mathrm{~s}^{-1}$ or better, utilizing a sector scan as narrow as $30^{\circ}$.

Easterbrook discusses the sensitivity of the method to vertical velocity and wind shear and to departures from linearity. He stresses the corresponding need to restrict the data field to low elevation angles (lower than $5^{\circ}$ ) and to a moderate extent both in range and azimuth.

Reexamining Eq. (4), where $\theta$ and $R$ now vary while $\phi$ does not, we see that when $x_{0}$ and $y_{0}$ are chosen equal to 0 , every term can be discriminated with the exception of $V_{7}$ (proportional to $w_{2}^{\prime}$ ) which partly contaminates $V_{4}$ and $V_{5}$ (divergence components) and partly $V_{3}$ (vertical velocity).

If the situation and/or data sample are such that $w$ does not vary appreciably with altitude, or if the analysis is restricted to low elevations, Eq. (3) can 
thus be used with eight independent terms. The method is more powerful than simple VAD or VARD since one obtains estimates of $u_{z}^{\prime}$ and $v_{z}^{\prime}$ which allow a check of $u_{0}$ and $v_{0}$. However, since the vertical velocity is not perfectly known, possible errors in divergence cannot be estimated either.

\section{c. Processing a volume}

If the radar records, as it often does, a series of conical scans with increasing elevation, and if the total acquisition time is short enough, there is no objection to processing simultaneously data having different elevation angles, within a narrow range of altitudes. In that case all nine terms of $V_{r}$ in Eq. (4) can, in principle, be discriminated. This is the most complete, and thus the most promising possibility. Compared to processing a more limited data sample, the volume analysis (this will be shortened to VVP, for Volume Velocity Processing) seems to offer the following avantages:

1) Horizontal mean velocity components $u_{0}$ and $v_{0}$ are retrieved without any contamination for any altitude or elevation angle.

2) The same can be said of the $u_{x}^{\prime}$ and $v_{y}^{\prime}$ terms, and hence of the horizontal divergence and, ultimately, of the vertical air velocity computed by integrating the continuity equation.

3) An estimate of the vertical component (nearly equal to the fall velocity of hydrometeors) is directly available.

4) Knowledge of derivatives along the vertical of all three velocity components allows a convenient control of the validity of the procedure by comparison between successive altitude slices.

Before applying the VVP method to real data, it is useful to test it on simulated samples in order to estimate its accuracy. This is done in the next two sections.

\section{VVP applied to simulated radar data: Error analysis}

The VVP technique, i.e., the analysis of data according to Eq. (4), has been implemented by use of a least-squares procedure. This has two main advantages: first, it largely eliminates possible biases due to uneven volume distribution of data points; and second, it provides a way to compute objectively standard deviations of estimates and thus to parameterize approximately the magnitude of the errors.

The main steps of the calculation are summarized in the Appendix. While the matrix inversion must be carried out using a double precision algorithm, no particular problem arose when retrieving the nine parameters.

The simulated data consist basically of seven conical scans with elevation varying between 0.5 and $24.5^{\circ}$ through $3.5^{\circ}$ steps. The gate separation is $400 \mathrm{~m}$, the azimuth step about $6^{\circ}$. The simulated wind field varies linearly along all three axes. No random-like error was superimposed to the linear field (recall that estimated standard deviations in a linear least-square adjustment do not depend on the actual data scatter). The adjustment was conducted on successive slices 500 or $1000 \mathrm{~m}$ thick, restricted to various parts of the total volume covered by the simulated file.

When every simulated data value for a given altitude slice was included in the analysis, the retrieved parameters were found identical (within truncation errors) to the simulated ones; hence, no comparative plot of these quantities will be given. However, since the discrimination between the wind field parameters is achieved through different variations with the polar coordinates, these coordinates $R, \theta$ and $\phi$ must be allowed to vary over a sufficient range in the sampled volume, otherwise the method fails. This evident restriction is illustrated in the following error analysis, which is based on numerical results obtained when VVP was applied to various fractions of the total simulated $V_{r}$ field. The following variations have been tested:

- Total number $N$ of data values (by choosing a larger range gate or azimuth coverage).

- Range coverage, limited to a maximum horizontal distance $r(\mathrm{~m})$.

- Elevation coverage $\Delta \phi$ (deg).

- Azimuth/area coverage, by restricting the analysis to a given side of a vertical plane located at a varying distance $p$ from the radar (Fig. 1a). This was preferred to a sector aperture variation because it is closer to the way horizontal echo coverage might be limited in real situations.

The results are briefly summarized below for all nine parameters of the wind field; recall they are empirical and represent orders of magnitude rather than exact formulas.

1) Every standard deviation is proportional to $\sigma_{0}=G(p / r) \sigma / \sqrt{N}$, where, $G(p / r)$ is a function of the area coverage when limited by a vertical plane, compared to the result obtained when processing a full cylinder of radius $r ; \sigma$ is the data standard deviation $\left(\mathrm{m} \mathrm{s}^{-1}\right)$, assumed constant, and $N$ is the number of data points.

$G(p / r)$ is plotted in Fig. 1b. Note that $G$ does not vary drastically as long as $p / r$ remains positive (i.e., when the volume exceeds half a circular cylinder); when $p / r$ becomes negative, however, $G$ increases very rapidly.

The $\sigma / \sqrt{N}$ dependence was expected in a situation where all data were assumed statistically independent. 
2) For the mean velocity components, we have

$$
\begin{aligned}
& \sigma\left(u_{0}\right) \approx \sigma\left(v_{0}\right) \approx 1.3 \sigma_{0}, \\
& \sigma\left(w_{0}\right) \approx 1.3 \sigma_{0} \times 90 / \Delta \phi .
\end{aligned}
$$

The elevation coverage dependence is not surprising in view of the nature of the $w$ term in Eq. (4).

3) For the horizontal derivatives we have

$$
\sigma\left(u_{x}^{\prime}\right) \approx \sigma\left(v_{y}^{\prime}\right) \approx \sigma\left(u_{y}^{\prime}+v_{x}^{\prime}\right) \approx 5 \sigma_{0} / r .
$$

The inverse dependence on maximum range is again consistent with the fact that it is easier to detect horizontal variations on large distances.

4) For the vertical derivatives we have

$$
\begin{aligned}
& \sigma\left(u_{z}^{\prime}\right) \approx \sigma\left(v_{z}^{\prime}\right) \approx 5 \times 10^{-3} \sigma_{0}, \\
& \sigma\left(w_{z}^{\prime}\right) \approx 3 \times 10^{-3} \sigma\left(w_{0}\right) .
\end{aligned}
$$

These errors do not seem to depend much on the thickness of the altitude slice, although such a dedependence would obviously appear for very small values of this thickness.

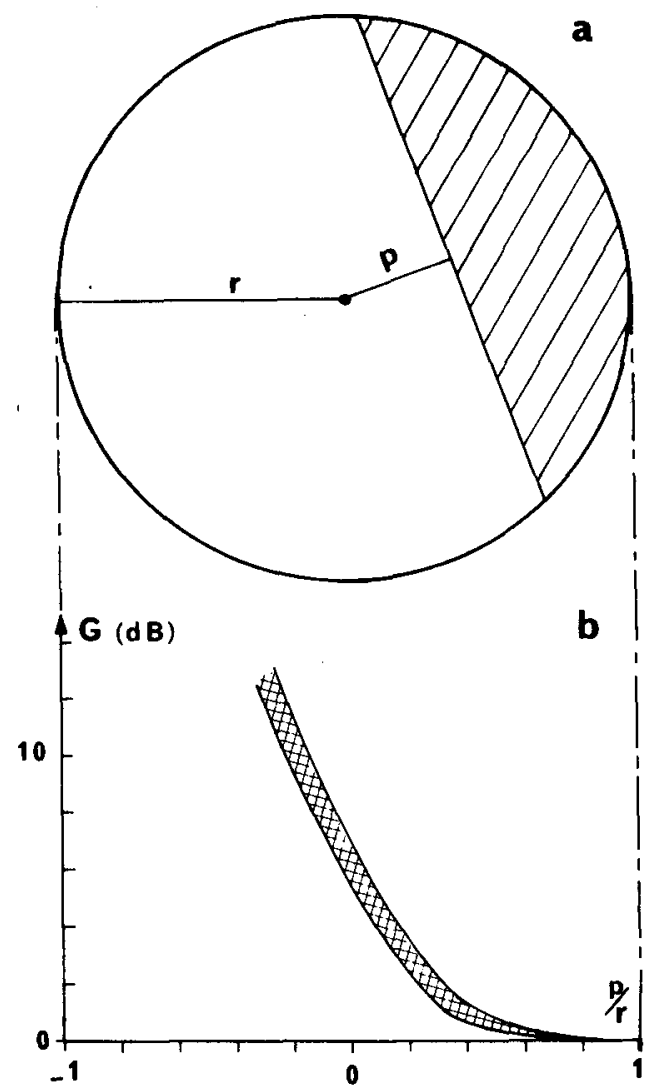

FIG. 1a. Sketch of the way the sampling volume is bounded by a vertical plane at distance $p$ from the radar; $p$ is positive when the radar itself is included in the sampling volume.

FIG. 1b. Variation with parameter $p / r$ of the empirical function $G$ describing the degradation of accuracy as $p / r$ decreases (see text).

\section{REMARKS}

A general rule is that the VVP method does not work properly unless $N$ reaches a few hundred, independently of any particular coverage limitation. To appreciate this number, it is useful to recall that modern Doppler radars are able to preprocess Doppler data in real time over many range gates and record currently, in a multiple conical scan, several $10^{4}$ to several $10^{5}$ radial velocity estimates within a few minutes.

Then a typical sample volume is likely to contain a few thousand data points. As a consequence, the errors computed using the above empirical formulae are very small. Indeed, it is natural that the larger the number of independent data, the better the resulting estimates ought to be.

In actual cases, however, the accuracy and significance of these estimates are limited by the inadequacy of the linear vector field approximation rather than random errors in velocity data, as will be shown below.

\section{Effect of nonlinearities in the wind field}

When applying the VVP technique to real data (see Section 5), it is found that the retrieval of the vertical velocity $w_{0}$ and its derivative $w_{z}^{\prime}$ is not completely successful. In looking for possible reasons for such a result, we can readily exclude an error in the computation code (since the analysis of simulated data yields good results) as well as errors in housekeeping information recorded by the radar (since some parameters are quite well-adjusted as confirmed by consistency with derivatives along the vertical). Therefore, the explanation must lie with the data field itself, i.e., some features of this field are not compatible with our hypotheses and specifically with a linear approximation.

As a check, we first introduce nonlinearities by adding higher order terms to the expansion of the wind field in Eq. (1) (see Appendix for details). These extra terms are again found in the expression of the radial velocity [Eq. (3)]. Although their dependence on polar coordinates differs from the original linear terms in Eq. (3), the possibility of contamination obviously exists. For example, a $w_{x^{2}}^{\prime \prime}$ contribution is proportional to $R^{2} \cos ^{2} \theta \cos ^{2} \phi \sin \phi$. Now, inasmuch as $\sin \phi$ has a nonzero mean value and $R^{2}$ can be written $\bar{R} R$, where the average $\vec{R}$ of $R$ in the analysis volume is nonzero, one might expect some contamination of $u_{x}^{\prime}$, the coefficient of which is $R \cos ^{2} \theta \cos ^{2} \phi$ with $\cos ^{2} \phi$ nearly equal to unity.

Before reporting the results, we state our terms of reference. We first indicate in Table 1, for the zeroth, first and second derivatives of the wind field, the orders of magnitude and maximum absolute values typical of the mesoscale (a few tens of kilometers to $100 \mathrm{~km}$ ). These values are inferred whenever possible 
TABLE 1. Typical orders of magnitude $m$ and maximum absolute values $M$ of mesoscale wind parameters.

\begin{tabular}{|c|c|c|c|c|c|}
\hline \multicolumn{2}{|c|}{ Order 0} & \multicolumn{2}{|c|}{ Order 1} & \multicolumn{2}{|c|}{ Order 2} \\
\hline Term & $\begin{array}{c}m, M \\
\left(\mathrm{~m} \mathrm{~s}^{-1}\right)\end{array}$ & Term & $\begin{array}{c}m, M \\
\left(10^{-4} \mathrm{~s}^{-1}\right)\end{array}$ & Term & $\begin{array}{c}\left(10^{-8}\right. \\
\left.m^{-1} s^{-1}\right)\end{array}$ \\
\hline \multirow[t]{2}{*}{$u_{0}, v_{0}$} & 10,30 & $u_{x}^{\prime}, u_{y}^{\prime} \ldots$ & 1,10 & $u_{x^{2}}^{\prime \prime}, u_{x y}^{\prime \prime}, \ldots$ & $0.5,3$ \\
\hline & & $u_{z}^{\prime} \ldots$ & 10,100 & $\begin{array}{l}u_{x z}^{\prime \prime}, u_{y z z}^{\prime \prime}, \ldots \\
u_{z^{2}}^{\prime \prime}, v_{z^{2}}^{\prime \prime}\end{array}$ & $\begin{array}{l}5,100 \\
100,500\end{array}$ \\
\hline \multirow{3}{*}{$w_{0}$} & 5,10 & $w_{x}^{\prime}$ & $0.1,1$ & $w_{x^{2}}^{\prime \prime}$ & $0.01,1$ \\
\hline & & $w_{z}^{\prime}$ & 5,80 & $w_{x z}^{\prime \prime}, w_{y z}^{\prime \prime}$ & $0.01,10$ \\
\hline & & & & $w_{z^{2}}^{\prime \prime}$ & 50,2000 \\
\hline
\end{tabular}

from previous work with Doppler radar on frontal systems (e.g., Browning and Harrold, 1970; Browning and Pardoe, 1973) and are consistent with results on actual data presented in the next section.

The possible occurrence of some of the maximum values listed in this table is very restricted; for example, high $w_{z}^{\prime}$ values and gradients are only present around melting layers; mesoscale gradients of $w_{x}^{\prime}$ can be large presumably in cases when this altitude undergoes significant space variations.

We shall now process the simulated file, where each nonlinear term in turn is added with increasing values of the second derivative, and look for these values of the latter which induce in any linear term, as retrieved through the VVP algorithm, a spurious change approaching a significant fraction $\left(\frac{1}{4}-\frac{1}{3}\right)$ of the order of magnitude specified for this term in Table 1. Here are the results:

- Terms such as $u_{x^{2}}^{\prime \prime}$ or $u_{x y}^{\prime \prime}$ contaminate terms such as $u_{0}, u_{z}^{\prime}, u_{x}^{\prime}$ and $w_{z}^{\prime}$. For $w_{z}^{\prime}$, the threshold is reached very early. For other terms, the results depend on the radius of the explored volume and deteriorate as this radius increases. With a $25 \mathrm{~km}$ radius, the threshold is $\sim 2 \times 10^{-8}$ $\mathrm{m}^{-1} \mathrm{~s}^{-1}$; with a $12.5 \mathrm{~km}$ radius, the threshold is above $3 \times 10^{-8} \mathrm{~m}^{-1} \mathrm{~s}^{-1}$.

- Terms such as $u_{x z}^{\prime \prime}$ contaminate slightly $w_{0}$ and severely $w_{2}^{\prime}$, for values as small as $5 \times 10^{-8}$ $\mathrm{m}^{-1} \mathrm{~s}^{-1}$. Other linear parameters stay unharmed until quite high values are reached $\left(10^{-6} \mathrm{~m}^{-1} \mathrm{~s}^{-1}\right)$.

- Terms such as $u_{z z}^{\prime \prime}$ have a negligible effect on their whole range of variation.

- Terms such as $w_{x^{2}}^{\prime \prime}$ contaminate seriously $u_{x}^{\prime}$ and $w_{z}^{\prime}$, from $10^{-8} \mathrm{~m}^{-1} \mathrm{~s}^{-1}$ only. This threshold value is given for a $25 \mathrm{~km}$ radius and decreases with increasing radius.

- Terms such as $x_{x z}^{\prime \prime}$ contaminate $u_{0}$ and $u_{z}^{\prime}$. This was expected since [see Eq. (3)] $w_{x}^{\prime}$ is compounded with $u_{z}^{\prime}$ in the expression of the radial velocity $V_{r}$. However, the deterioration is not severe for values lower than $3 \times 10^{-8} \mathrm{~m}^{-1} \mathrm{~s}^{-1}$.

- The $w_{2^{2}}^{\prime \prime}$ term has a negligible effect on its whole range of variation.
For each of these simulations, we have also carried out a standard VAD calculation. The comparison between VVP and VAD results shows that, in cases where nonlinear terms contaminate parameters which can be retrieved by both methods (i.e., mainly the influence of $u_{x^{2}}^{\prime \prime}$ on $u_{0}$ and $u_{x}^{\prime}$ ), the results are fairly similar in terms of threshold, and the conclusion, as noted also by Easterbrook (1975) for surface methods, is that one should try to limit the horizontal extent of the analyzed sample. Let us mention that in the VAD case this means that for higher altitudes one should use higher elevations; but this choice is precluded due to contamination of divergence by vertical velocities, unless this quantity can be estimated independently.

On the other hand, the VVP formulation indicates that we may be able to estimate other interesting parameters such as $w_{0}$ and $w_{z}^{\prime}$; while $w_{z}^{\prime}$ can be severely damaged by higher scale nonlinearities, their effect on $w_{0}$ was always found smaller than $1 \mathrm{~m} \mathrm{~s}^{-1}$.

This is not actually very surprising, because higher order derivatives introduce faster variations with radial distance, whereas the coefficient of $w_{0}$ does not depend on $R$ at all [see Eq. (4)].

The actual wind field may also differ from a linear one due to small-scale irregularities rather than to large-scale changes. We must now investigate this alternate possibility.

Even so-called stratiform situations quite often present a cellular structure with typical scales, as observed on radar reflectivity patterns, of the order of a few kilometers to a few tens of kilometers. Whenever this structure also exists in the velocity field, it is certain to influence the success of processing techniques assuming linearity. Evidently, the divergence computed around a volume the order of a "cell" is likely to differ markedly from and probably much exceed in magnitude the mesoscale divergence, which can only in principle be estimated around a volume much larger than the one typical of finestructure. Thus one particular effect of small-scale irregularities is to create a spurious divergence field, the value of which decreases as the radius increases. The contamination of vertical velocity by such phenomena appears to be a possibility.

In order to test this hypothesis, we introduce in the simulated data file a wind component varying sinusoidally with horizontal coordinates, superimposed on the linear wind field, and investigate the result when its amplitude $u_{s}$ and wavelength $\lambda$ vary. Strong effects appear on $w_{0}$ as well as on the horizontal derivatives, and they are most intense when the spurious wave has a zero amplitude above the radar. The magnitude of the contamination of $w_{0}$ is of the order of $u_{s}$ (that would be, in the atmosphere, up to 1-3 $\mathrm{m} \mathrm{s}^{-1}$ ). The effect on divergence depends to a large extent on the divergence of the wave $d_{s}=u_{s}$ $\times 2 \pi / \lambda_{s}$ and the ratio $\lambda / r$ of its wavelength to the 
analysis radius. When this ratio becomes smaller than 0.5 , the wave contribution to the divergence is typically $0.1 d_{s}$. The same applies to the other differential properties of the wind field.

Quantities retrieved by the VAD technique fluctuate widely with the analysis radius. These fluctuations are averaged to a large extent by the volume technique, while part of them is expressed in the contamination of $w_{0}$.

Summarizing the results of this section, we can see that on the whole the effect of larger scale irregularities is not likely to cause too much concern when studying mesoscale features (this goes for VAD as well as VVP). On the other hand, due to the combined effects of large-scale and small-scale departures from linearity, the retrieval of $w_{0}$ and especially $w_{z}^{\prime}$ is likely to suffer significant errors. In the following section, we test this on experimental data.

\section{VVP applied to actual radar data}

Data recorded with the RONSARD I Doppler radar on 2 April 1976 were processed by the VVP technique. RONSARD, a dual-Doppler C-band system, was developed in France over the period 1973-77; indeed, the first radar was first operated in the field in the spring of 1976, and the data considered here were recorded within the first few hours of operation. As a result there were a number of drawbacks in the observations. For example, there was neither good reflectivity data nor ground echo rejection that day. The velocity values, however, are considered reliable on the whole. A few ambiguities (the selected ambiguous velocity range was $\pm 20 \mathrm{~m} \mathrm{~s}^{-1}$ ) were removed using an algorithm very similar to the one described by Ray and Ziegler (1977).

On April 1976 RONSARD was located at Magnyles-Hameaux, $20 \mathrm{~km}$ southwest of Paris. The scanning mode was a sequence of full conical scans, with elevation increasing from $0.5^{\circ}$ to $24.5^{\circ}$ through $3.5^{\circ}$ steps. The range gates were contiguous and $200 \mathrm{~m}$ wide and the maximum range was $40 \mathrm{~km}$; a full volume exploration took about $5 \mathrm{~min}$, for a total of about $3.1 \times 10^{5}$ data gates.

The echo structure observed near the end of afternoon consisted of a weak reflectivity band about $40 \mathrm{~km}$ wide, with echo top near $6.5 \mathrm{~km}$, associated with a slowly moving cold front. We have selected an exploration near 1710 GMT, when the band was approximately on top of the radar. The VVP method was applied to successive altitude slices $250 \mathrm{~m}$ thick; the maximum horizontal range was $15 \mathrm{~km}$. Typical results are summarized on Fig. 2, where vertical profiles of the horizontal and vertical velocities are plotted, together with their derivatives with altitude, as determined by the VVP algorithm.

The horizontal wind magnitude and direction $V_{h}$ and $\alpha$ deserve little commentary. They offer reasonable continuity with altitude. The picture offered by the vertical derivatives $V_{h z}^{\prime}$ and $\alpha_{z}^{\prime}$ is on the whole very satisfactory. In some regions the agreement is excellent, indeed, in the sense that there is perfect continuity in altitude between broken lines describing

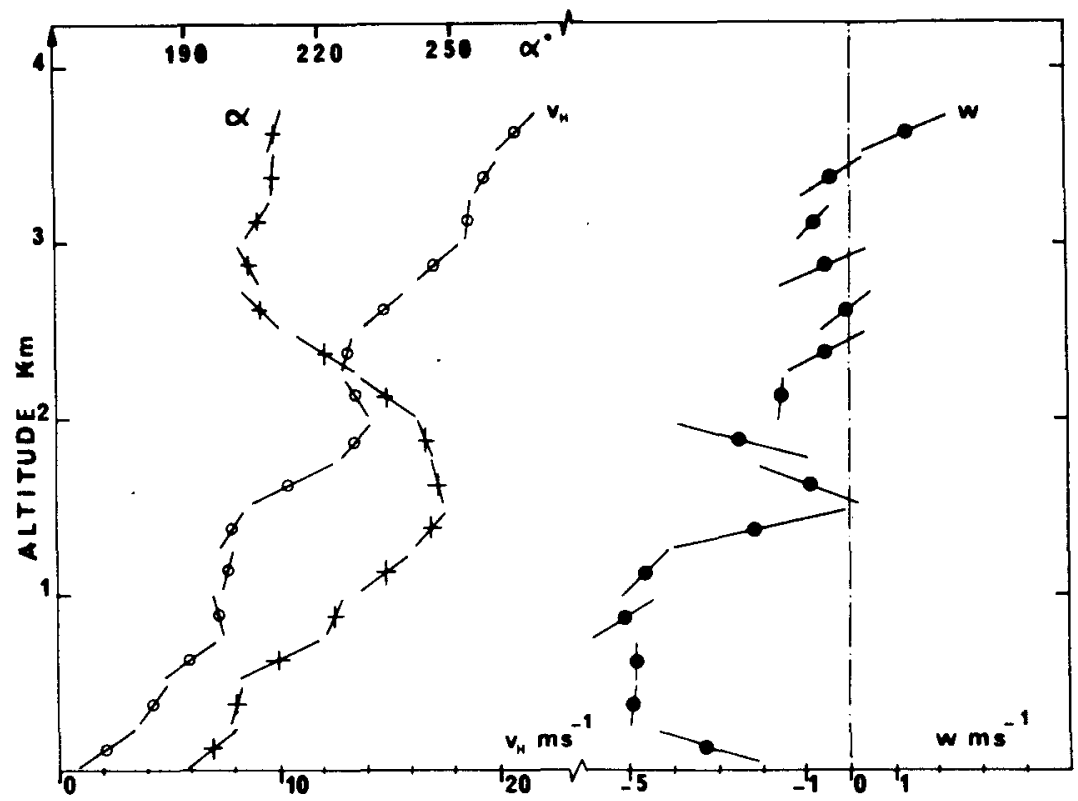

FIG. 2. Vertical profiles of velocity components (module $V_{h}$ and azimuth $\alpha$ of horizontal component, vertical component $w$ ) obtained when applying the VVP procedure to a set of RONSARD data at 1710 GMT 2 April 1976. The profiles are made of broken lines drawn according to derivatives retrieved by VVP. Radial velocity data above $4 \mathrm{~km}$ were very scarce and could not be accurately processed. 


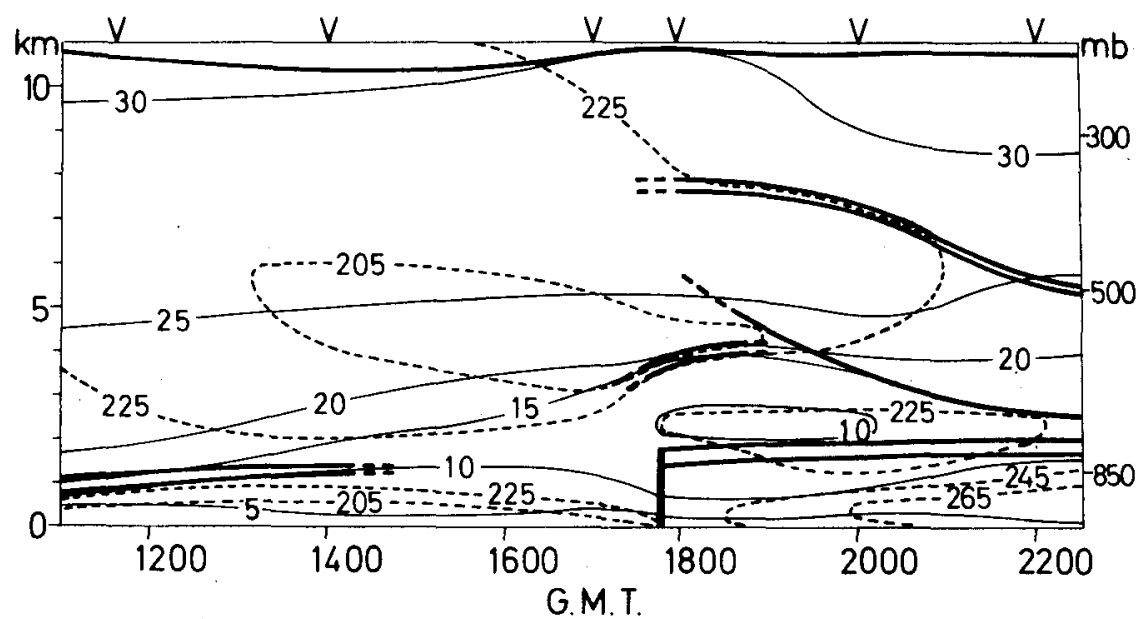

FIg. 3. Time-height section of horizontal wind magnitude and direction above Magny-lesHameaux on 2 April 1976, from local soundings (launch time is indicated by arrows at the top of diagram). The frontal zone and stable layers are indicated by thick lines, as well as main air mass boundaries.

the linear variation adjusted across each altitude slice. In other regions, although this continuity is not perfectly achieved, the derivatives are far from aberrant. Possible distortions on $u_{z}^{\prime}$ and $v_{z}^{\prime}$ due to large values of $w_{x}^{\prime}$ and $w_{y}^{\prime}$ (see Section 2) do not appear to be present.

More serious difficulties arise when discussing the vertical $w_{0}$ and $w_{2}^{\prime}$ profiles. The $w_{0}$ values are sometimes anomalous (especially the near zero or weakly positive values above $2 \mathrm{~km}$; the lowest altitude slice was probably contaminated by ground echoes). Similarly, the $w_{z}^{\prime}$ values in the upper part of the profile are not in general compatible with the trend indicated by the $w_{0}$ values. Nevertheless, the $w_{0}$ overall altitude variation, exhibiting values near $-5 \cdot \mathrm{m} \mathrm{s}^{-1}$ in the lowest kilometer, increasing to near $-0.5 \mathrm{~m} \mathrm{~s}^{-1}$ values above $2 \mathrm{~km}$, is entirely consistent with the physical situation $\left(0^{\circ} \mathrm{C}\right.$ level near $1500 \mathrm{~m}$ above ground, weak rain observed at radar site).

Looking at vertical profiles of $u_{x}^{\prime}$ and $v_{y}^{\prime}$ (not shown), it is apparent that some of the most erratic values found for $w_{z}^{\prime}$ are correlated with steep variations of $u_{x}^{\prime}$ or $v_{x}^{\prime}$ with altitude. This is in agreement with the contamination effect noted previously on $w_{z}^{\prime}$ for large magnitudes of terms such as $u_{x_{2}}^{\prime \prime}$. Also, inspection of the raw data on PPI displays indicates that departures from linearities with $1-2 \mathrm{~m} \mathrm{~s}^{-1}$ amplitudes and 5-10 km scales are present in the data set; this accounts for the scatter of $w_{0}$ values.

The meteorological situation on 2 April 1976 in western Europe was investigated using extra information from synoptic soundings and from radiosondes released at the radar site at intervals of $2-3 \mathrm{~h}$. The full results of this investigation are being prepared for separate publication. They establish that frontolysis was taking place due to changes of the circulation at middle and upper tropospheric levels. Fig. 3 shows a height-time diagram of the wind direction contours above the radar site. The frontal zone is indicated as well as the main air mass boundaries and the stable layers. The meteorological analysis shows how the subsidence of a relatively cold, dry air mass contributed to the local disappearance of the front after 1800 GMT. Recall that the situation was evolving rather rapidly and Fig. 3 may bear no relation to an existing space-height diagram. The ground trace of the front did pass the radar site at Magny-lesHameaux, with probably some undulations, around 1745 , and from then on the local system did not move appreciably until it disappeared.

There is no doubt that finestructure elements were present in the velocity field at every altitude in the vicinity of the surface front; again this accounts for the poor retrieval of vertical velocity and derivatives about 1710 GMT shown on Fig. 2. The results (not shown) for $w_{0}$ and $w_{z}^{\prime}$ about 1900 , when convection activity was decreasing, are significantly less scattered.

One expects that the circulation will differ on each side of the surface front; this suggests that we apply a VVP procedure to half-cylinders separated by a vertical plane parallel to the surface front (i.e., very nearly southwest-northeast). In such cases, we have seen that $u_{0}$ and $v_{0}$ values are only retrieved for $x_{0}=y_{0}=0$; on the other hand, every other quantity is representative of the analyzed volume and can be assigned to the barycenter of this volume.

The result is illustrated on Figs. $4 \mathrm{a}$ and $4 \mathrm{~b}$ by time-height diagrams showing the divergence fields on each side of the sèparating surface. In the present discussion, we leave aside the features observed prior to $1800 \mathrm{GMT}$ : these variations are associated to the vicinity of the still somewhat active front, and will be commented on elsewhere. After 1800, the structure of the divergence field becomes more regular. The 
main feature is the presence aloft of a convergent zone $\sim 1 \mathrm{~km}$ thick, the altitude of which increases with time.

Looking back at Fig. 3, we see that this feature is present between the front and a temporary stable layer located 2-2.5 $\mathrm{km}$ above the frontal surface. Such a convergence could possibly be induced by the arrival of the frontal boundary, and last long enough to generate the slight precipitation which allowed radar observations.
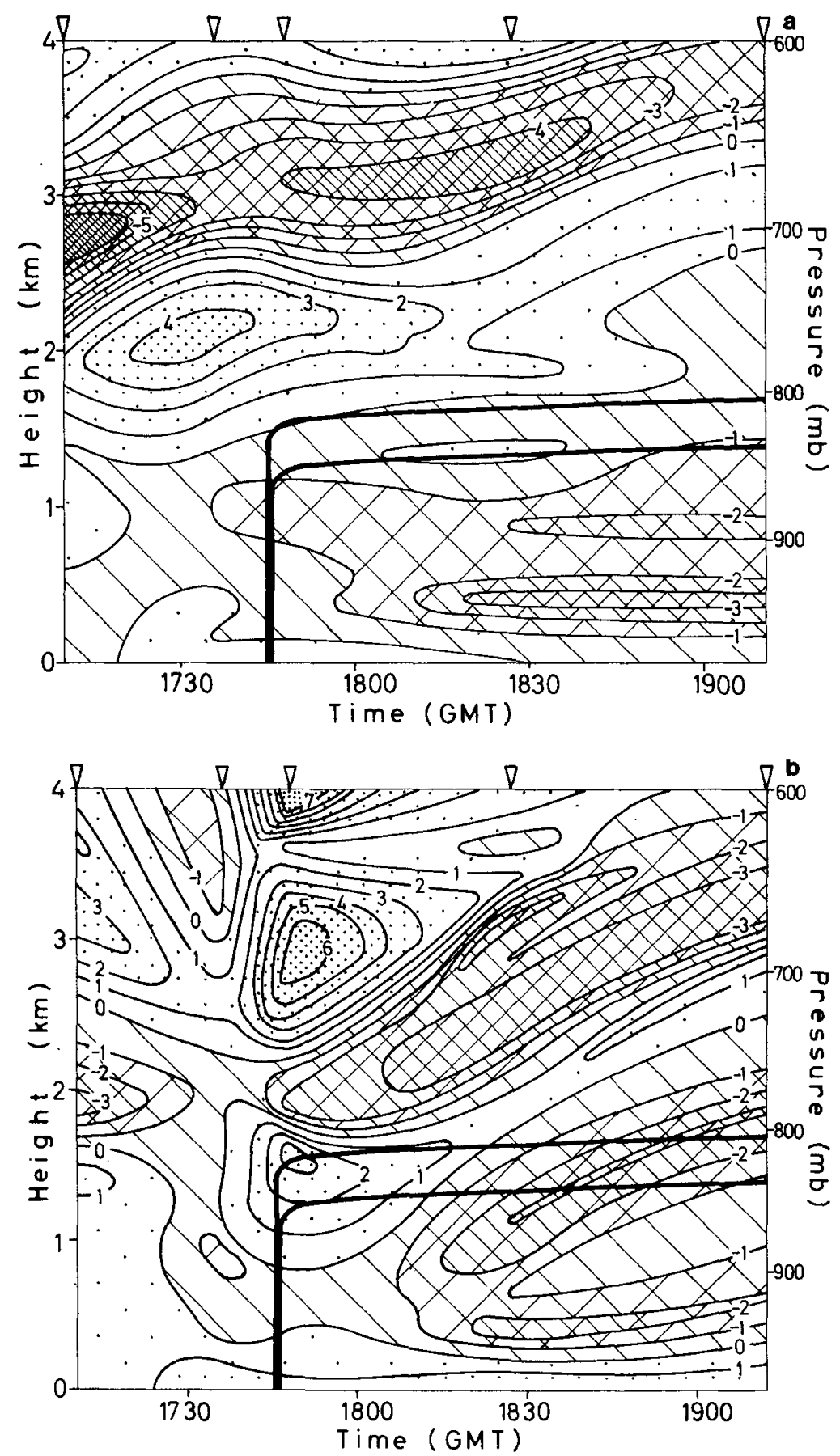

Frc. 4. Time-height section of the divergence field obtained from RONSARD data through the VVP technique, applied to each half of a circular cylinder with $20 \mathrm{~km}$ radius and $250 \mathrm{~m}$ thickness. The contour step is $10^{-4} \mathrm{~s}^{-1}$. (a) northwest of the radar; (b) southeast of the radar. 
It is of obvious interest to know whether this feature has any spatial extent. Again, since the situation changes rapidly (and the mean wind blows nearly parallel to the surface front), no pertinent information on this particular point can be derived reliably from Fig. 3 taken alone. The VVP procedure, however, does bring the elements of an answer. Fig. 4 shows that the divergence behavior on either side of the separating plane is quite similar after 1800 . In particular, the same convergent zone aloft undoubtedly is found on both diagrams. Since the volumes for which the divergence is computed have barycenters separated by $\sim 15 \mathrm{~km}$ horizontally, this gives us an indication of the minimal extent of the convergence band along a northwest-southeast line. Moreover, the time-height divergence diagrams, although similar, are not identical: the convergent band, as well as slightly divergent zones above and below, is strongly tilted toward the southeast at about 1800 GMT, then levels off and becomes horizontal at 1900. This is in good agreement with the general trend of the stable layer overhanging the front as well as the surrounding wind directions contours on Fig. 3. Therefore, the organization of such features in space is, to some extent, correctly depicted by the time-height diagram.

\section{Discussion and conclusion}

We have shown how every method proposed to process single-Doppler radar data, based on linear properties of the wind field, may be considered as a restricted version of a general scheme where the linearity assumption is taken advantage of in a whole volume. We have further indicated what appeared to us to be the less severe restrictions in order that the more general algorithm be applicable. Having then tested this method both on simulated and actual data, we are now able to present a balanced evaluation of the VVP technique.

First, let us mention that the procedure is not unduly clumsy when compared to others. Also, the concept of volume sampling tends to improve versatility, since the way data are processed becomes completely independent of the way and order in which they have been taken.

The VVP procedure is theoretically able to retrieve the vertical velocity of hydrometeors and their vertical gradients, $w_{0}$ and $w_{z}^{\prime}$; the error analysis suggests that both parameters can be obtained with excellent accuracy. Unfortunately, they turn out to be sensitive to departures from linearity and wind field irregularities, to such an extent that the $w_{0}$ and $w_{z}^{\prime}$ estimates cannot be relied on.

Typical errors in $w_{0}$ due to these phenomena are of the order of $1-2 \mathrm{~m} \mathrm{~s}^{-1}$. This is larger than vertical air velocities currently encountered in frontal precipitation zones. On the other hand, it is good enough to insure that no serious contamination to the di- vergence is likely to occur. Thus, it still seems that the VVP procedure make it possible to retrieve an unbiased divergence by itself, without the necessity of extra information such as direct measurements of the vertical velocity or indirect estimates of this quantity from reflectivity values.

We find that departures from linearity in the actual wind field constitute the basic limitation of the VVP method. Nonlinearities on scales larger than the analyzed volume primarily induce errors in the vertical shear values. Errors in the characteristics of the horizontal wind field affect VVP results in a manner similar to their affect on VAD results. However, since the VVP is able to deal with higher elevation angles than VAD (because it provides for the vertical velocity), it is possible for a given altitude range to process data in a cylinder of minimum radius, so as to avoid part of the nonlinear effects.

Small-scale irregularities (waves, finestructure of the wind field) also have an effect on the performance of VVP, particularly concerning the retrieval of $w_{z}^{\prime}$ and $w_{0}$. We stress, however, that in this respect VAD and VVP function differently. The divergence computed by a VAD technique, with or without leastsquares adjustment, does not actually depend on the linear hypothesis. With the exception of the contamination by the vertical velocity of hydrometeors, it is an exact quantity. On the other hand, quantities computed through a volume technique such as VVP do assume linearity, with the result that the finestructure of the wind field is low-pass filtered. We have not investigated the detailed effect of this filtering process in space; nonetheless, it is clear that contributions from features with characteristic scales smaller than the circle diameter are substantially reduced. This goes for the mean horizontal wind as well as differential quantities. In other words, results from the VVP procedure tend to be more representative of larger scale (i.e., mesoscale) features. It is quite easy to vary the cylinder radius, in order to verify that in a given range the influence of radius on the results becomes small: whenever such a range exists, it corresponds to the case where small-scale irregularities are being smeared out, while large-scale nonlinearities have not yet taken over. It is also possible to control the validity of a VVP adjustment by checking the continuity of $u_{0}$ and $v_{0}$ vertical profiles using the retrieved values of $u_{z}^{\prime}$ and $v_{z}^{\prime}$; continuity of the $w_{0}$ profile using $w_{z}^{\prime}$ values would indicate that there is very little small-scale wind structure in the analyzed volume.

Finally, one can take advantage of the very large number of data available in a volume method, to apply separately the procedure to fractions of a circular cylinder. Although the possibilities for doing this are obviously limited, it may be very useful when the presence of a sharp discontinuity within the volume under study is suspected. We have shown 
that performing a VVP analysis on contiguous halves of a circular cylinder made it possible to get an idea of the spatial structure of differential quantities which characterize the wind field. This may be seen as an alternative and instructive method of dealing with large-scale nonlinearities.

The best way to determine experimentally the wind field in precipitating zones is certainly to use several Doppler radars in coordinated operation. Experiments involving such a setup have already been conducted, and there will be more. Field work with single radar, however, will obviously continue for a long time due to practical considerations. Besides, there are physical situations where a single radar is able to gather most of the essential, dynamical information. Even using several radars, the accuracy of direct vertical velocity restitution is moderate to poor especially at low levels; for example, in nearly stratiform conditions, it will still be best to work from accurate, unbiased horizontal divergences.

In this context, efforts to clarify and improve single-Doppler processing methods are certainly justified; for example, Breger (1977) has carried out a least-squared adjustment on circles, assuming the wind consists of a linear field superimposed to a wave structure. He was thus able to identify such a wave with a sensitivity much improved with respect to Harris (1975). As for the VVP method which has been discussed in this paper, it offers a number of specific advantages, and we believe that such a method may help to make the best use of single-Doppler radar measurements in meteorological research.

Acknowledgments. This research was supported by CNRS, under Grant INAG/ATP RA/76. The RONSARD program has been financed by CNET (Centre National d'Etudes des Télécommunications) and DGRST (Délégation Générale à la Recherche Scientifique et Technique). Helpful comments by Dr. D. Ramond are gratefully acknowledged.

\section{APPENDIX}

\section{Details of VVP Implementation and Simulation}

\section{Implementing the linear-least squares procedure}

Assuming the wind field is linear in a given volume [Eq. (1)] led us to write that the measured radial velocity $V_{r}$ is a linear function $F$ of wind components [Eq. (4)]. We now rewrite this equation for convenience as

$$
F=\sum_{j=1,9} \frac{\partial F}{\partial u_{j}} u_{j}
$$

where the $u_{j}$ (wind field parameters) and $\partial F / \partial u_{j}$ (coefficients depending on polar coordinates) are given in Table A1.
TABLE A1. Factors entering the expression of $F$.

\begin{tabular}{lll}
\hline$j$ & \multicolumn{1}{c}{$u_{j}$} & \multicolumn{1}{c}{$m_{j}=\partial F / \partial u_{j}$} \\
\hline 1 & $u$ & $\cos \theta \cos \phi$ \\
2 & $v$ & $\sin \theta \cos \phi$ \\
3 & $v_{\prime}^{\prime}$ & $\sin \phi$ \\
4 & $u_{x}^{\prime}$ & $R \cos ^{2} \theta \cos ^{2} \phi$ \\
5 & $v_{y}^{\prime}$, & $R \sin ^{2} \theta \cos ^{2} \phi$ \\
6 & $u_{y}^{\prime}+v_{x}^{\prime}$ & $\sin \theta \cos ^{\prime} \cos ^{2} \phi$ \\
7 & $w_{z}^{\prime}$ & $\sin \phi\left(R \sin \phi-z_{0}\right)$ \\
8 & $u_{z}^{\prime}$ & $\cos \theta \cos \phi\left(R \sin \phi-z_{0}\right)$ \\
9 & $v_{z}^{\prime}$ & $\sin \theta \cos \phi\left(R \sin \phi-z_{0}\right)$ \\
\hline
\end{tabular}

The least-square technique is implemented by minimizing the expression

$$
H=\sum_{i} \frac{\left(V_{r i}-F_{i}\right)^{2}}{\sigma_{i}{ }^{2}},
$$

where the sum is carried over all experimental values. We assume here that the variance of each velocity measurement is the same (i.e., $\sigma_{i}=\sigma$ ) and write that in order for $H$ to be a minimum, its derivatives with respect to the $u_{j}$ must vanish, i.e.,

$$
\frac{\partial H}{\partial u_{k}}=-\frac{2}{\sigma^{2}} \sum_{i}\left(V_{r i}-\sum_{j} \frac{\partial F_{i}}{\partial u_{j}} u_{j}\right) \frac{\partial F_{i}}{\partial u_{k}}=0 .
$$

We thus have to solve the linear system

$$
\sum_{j}\left(\sum_{i} \frac{\partial F_{i}}{\partial u_{j}} \frac{\partial F_{i}}{\partial u_{k}}\right) u_{j}=\sum_{i} \frac{\partial F_{i}}{\partial u_{k}} V_{r i}
$$

The matrix of the system $M_{j k}=\sum_{i} m_{j} m_{k}$ is obtained readily using the $m_{j}$ expressions given in Table 1 . In order to solve this system for the $u_{j}$, we simply have to compute by standard methods the inverse matrix $\mathbf{M}^{-1}$. We then obtain

$$
u_{j}=\sum_{k} M_{j k}^{-1} \sum_{i} \frac{\partial F_{i}}{\partial u_{k}} V_{r i} .
$$

We now assume that the $V_{r i}$ values are changed by an amount $\delta V_{r i}$. The resulting $u_{j}$ values will then be changed by corresponding amounts $\delta u_{j}$; due to the linearity of the lationship, one has

$$
\delta u_{j}=\sum_{k} M_{j k}^{-1} \sum_{i} \frac{\partial F_{i}}{\partial u_{k}} \delta V_{r i}
$$

Consider now that the $\delta V_{r i}$ are independent, experimental errors and assume their distribution to be Gaussian. Since $u_{j}$ is a sum of Gaussian independent variables, we can write immediately its variance

$$
\sigma^{2}\left(u_{j}\right)=\left(\sum_{k} M_{k j}^{-1} \sum_{i} \frac{\partial F_{i}}{\partial u_{k}}\right)^{2} \sigma^{2} .
$$


The coefficient of $\sigma^{2}$ on the right-hand side of Eq. (A6) can be expanded to give

$$
\sigma^{2}\left(u_{j}\right)=\left(\sum_{k l} M_{j k}^{-1} M_{j l}^{-1} M_{k l}\right) \sigma^{2} .
$$

Most terms of the $\sum_{k l}$ sum vanish and one finally obtains

$$
\sigma^{2}\left(u_{j}\right)=M_{j j}^{-1} \sigma^{2}
$$

\section{Further characteristics of the simulated data file}

The simulated radial velocity was computed according to Eq. (2) with

with

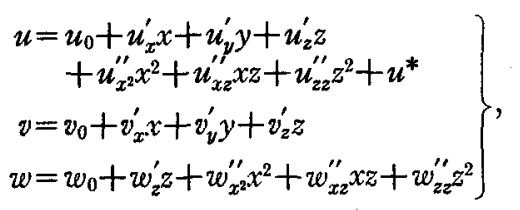

$$
u^{*}=u_{s} \cos \left[2 \pi\left(x-x_{1}\right) / \lambda\right]
$$

The linear coefficients $\left(u_{0}, u_{x}^{\prime}, u_{y}^{\prime}, u_{z}^{\prime}, v_{0}, v_{x}^{\prime}, v_{y}^{\prime}, v_{z}^{\prime}, w_{0}, w_{z}^{\prime}\right)$ were given several sets of values typical of mesoscale observations. The nonlinear coefficients $\left(u_{x^{2}}^{\prime \prime}, u_{x y}^{\prime \prime}, u_{z z}^{\prime \prime}\right.$, $\left.w_{x,}^{\prime \prime}, w_{x z}^{\prime \prime}, w_{z z}^{\prime \prime}\right)$ were assigned in turn a sequence of increasing values.

Similarly, values of $u_{2}, x_{1}$ and $\lambda$ in Eq. (A10) were varied to simulate a small-scale (wavelike) irregularity.

The simulated data were processed identically to actual data except that a plane earth was assumed.

\section{REFERENCES}

Battan, L. J., 1977: Rain resulting from melting ice particles. J. Appl. Meteor., 16, 595-604.

Breger, G., 1977: Etude par radar Doppler des mouvements atmospheriques au voisinage d'un front froid. Thèse de 3ème cycle, Université de Paris VI, 108 pp.

Browning, K. A., and T. W. Harrold, 1970: Air motion and precipitation growth at a cold front. Quart. J. Roy. Meteor. Soc., 96, 369-389.

Browning, K. A., and C. W. Pardoe, 1973: Structure of low level jet streams ahead of mid-latitude cold fronts. Quart. J. Roy. Meleor. Soc., 99, 619-638.

--, and R. Wexler, 1968: The determination of kinematic properties of a wind field using Doppler radar. J. Appl. Meleor., 7, 105-113.

Caton, P. G., 1963: The measurement of wind and convergence by Doppler Radar. Preprints Tenth Weather Radar Conf., Washington, DC, Amer. Meteor. Soc., 290-296.

Easterbrook, C. C., 1975: Estimating horizontal wind fields by two dimensional curve fitting of single Doppler radar measurements. Preprints 16th Radar Meteorology Conf., Houston, Amer. Meteor. Soc., 214-219.

Harris, F. I., 1975: Motion field characteristics of the evaporative base region of a stratiform precipitation layer as determined by ANDASCE. Preprints 16th Radar Meteorology Conf., Houston, Amer. Meteor. Soc., 225-230.

Lhermitte, R. M., and D. Atlas, 1961 : Precipitation motion by pulse Doppler. Preprints Ninth Weather Radar Conf., Kansas City, Amer. Meteor. Soc., 218-223.

Peace, R. L., Jr., R. A. Brown and H. G. Camnitz, 1969: Horizontal motion field observations with a single pulse Doppler radar. J. Atmos. Sci., 26, 1096-1103.

Ray, P. S., and C. Ziegler, 1977 : Dealiasing first-moment Doppler estimates. J. Appl. Meteor., 16, 563-564. 